\title{
Development of an UWB based Indoor Positioning System
}

\author{
$1^{\text {st }}$ Marcell Molnár \\ Systems and Control Lab \\ Institute for Computer Science and Control \\ Budapest, Hungary \\ marcell.molnar@sztaki.hu
}

\author{
$2^{\text {nd }}$ Tamás Luspay \\ Systems and Control Lab \\ Institute for Computer Science and Control \\ Budapest, Hungary \\ tluspay@sztaki.hu
}

\begin{abstract}
The demand for precise indoor positioning is increasing nowadays, since traditional methods, like GPS, cannot be used in indoor environments. Lots of technologies exist that can be used for such purposes, having their own advantages and disadvantages when it comes to positioning. However, it seems that UWB is the most favorable, having the possibility to achieve the best accuracy due to its spectral features. Although, in the past years several companies appeared as a UWB chip and antenna manufacturer providing out-of-the-box solutions, most of them only have chips or demonstration kits to purchase. The paper shows the development and testing of an UWB indoor positioning system using commercially available hardware and specific software elements in order to allow flexibility required for research purposes.

Index Terms-UWB, indoor positioning, ToF, SDS-TWR
\end{abstract}

\section{INTRODUCTION}

The paper documents the development of an UltraWideBand (UWB) based indoor positioning system [1]. The positioning system's goal is to be able to localize the position of a device, usually attached to a moving object such as unmanned aerial vehicles (UAV) or autonomous ground vehicles, and also widely used in areas of robotics, Industry 4.0 or other location-based services. The need for knowing the exact position of a moving object is not new; the Global Positioning System (GPS) has existed for 40 years and it can provide a precise location at almost any point on the Earth [2]. But when it comes to indoor positioning, the GPS is not applicable because of the attenuation of the received signal strength. Therefore, other technologies have to be applied.

Several technologies can be used in indoor environments, like vision or radio based systems. Although vision based systems can achieve sub-millimeter accuracy, they cannot be used under visually limited conditions. On the other hand, radio based solutions can be used in any visual conditions, and can provide centimeter to millimeter accurate position. Among radio technologies, UWB technology has the advantage of high data rate at low power consumption [3]. According to the Federal Communications Commission and International Telecommunication Union Radio communication Sector (ITU-R), UWB is a radio technology that has a bandwidth greater than $500 \mathrm{MHz}$ or covering more spectrum than $20 \%$ of its carrier frequency [4]. Because of the wide spectrum, in time domain it has very narrow pulses, which is ideal for measuring propagation time between nodes.

In the terminology of UWB, tag refers to the device which has an unknown position and has to be localized. For this purpose it is recommended to use at least 4 anchors, which are devices installed in a fix and known position [1]. Many approaches are available in the literature to calculate the tag's position using Time Difference of Arrival (TDoA) [5], or Time Sum of Arrival (TSoA) [6], for instance. These methods require precise synchronization of the anchors' clock [7], [8]. One can also use instead the Time of Flight (ToF) with Symmetrical Double-Sided Two-Way Ranging (SDSTWR) communication scheme [9], which does not require any clock synchronization. Double Two-Way Ranging (DTWR) method [10], [11] is also an alternative solution, which can give faster measurements than the SDS-TWR.

The ToF method provides distances from the anchors to the tag. The next step is to calculate the tag's position from the distances and the known anchor positions and there are three main approaches to do it [12]. The first main group are the statistical methods, based on a certain hypothesis about the measurements and the position of the tag. These information are related with a probabilistic model and linear approximations or iterative numerical methods are applied for obtaining the position [13]. These techniques can provide an optimal estimation of the position. However, the solution can not be expressed in a closed form (i.e. iterative numerical methods are needed) and a previous ( $a$ priori) estimation of the position is also required. Algebraic methods form the second group, based on the equations relating the devices' positions with the distances [14]. In contrast with the statistical methods, these approaches do not require any previous estimations of the position and have a predetermined execution time, hence can be used in real-time applications. However, it is the easiest to solve the problem with the numerical methods, which is the third main class for computing position [15]. The main advantage of numerical methods is that only a relation between the unknown parameters and the measurements is needed. The solution is usually obtained through a nonlinear optimization, mostly by minimizing a cost function. Numerous solvers exist to solve this problem, therefore these methods are the easiest to implement.

The paper's goal is to provide a detailed guideline on the development of an UWB indoor positioning system, presenting the hardware, software and mathematical aspects of the system. First the system components are introduced in Section II. A detailed explanation on the system properties, like communication scheme and position calculation, can be found in Section III. Considerations on the variance of the measurements are also taken into account in the same section. It is a major problem how to place the anchors and measure the positions of the installed anchors, which are presented in Section IV. Lastly, the system validation can be found in V. 


\section{SySTEM COMPONENTS}

The section describes the hardware and software components of the system, with the overall setup depicted in Figure 1 .

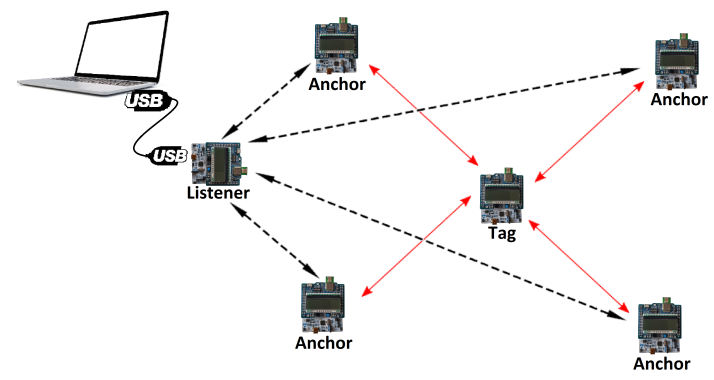

Fig. 1. The setup of the proposed system

\section{A. Hardware Elements}

The tag and anchor devices consist of two main parts as seen in Figure 2. An STM32 Nucleo-64 microcontroller is used as the main chip, while the UWB communication is performed via a DWM100 module by DecaWave [16]. The main chip implements a state machine that drives the DWM1000 module to perform the SDS-TWR method (see Section III-A).

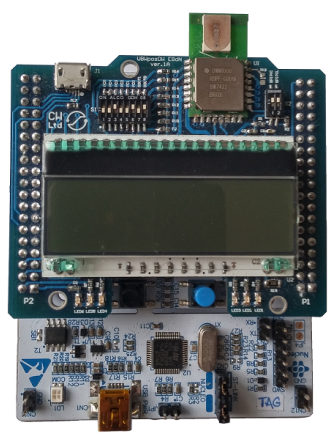

Fig. 2. The used board and UWB chip

\section{B. Software Elements}

There are two independent subsystems in the proposed system (see Figure 1). First, a C-based program runs on the anchors and on the tag which is responsible for the distance measurements. Second, the calculation of the position is performed on a PC and its code is written in Python.

a) Microcontroller Software: Initially the DWM1000 chip was supplied with a base code from the manufacturer. This basic firmware implements a state machine and an SPI based communication with the chip. Porting to the used microcontroller and minor modifications were necessary, since the usage of another microcontroller. Therefore, the pinout of the chip has been changed, in order to make a connection between the microcontroller and the DWM1000 chip. Also, the LCD display, additional LEDs and DIP switches had to fit on the board outputs.

The listener device has a special role in the system configuration (see Figure 1): it only listens to the messages,

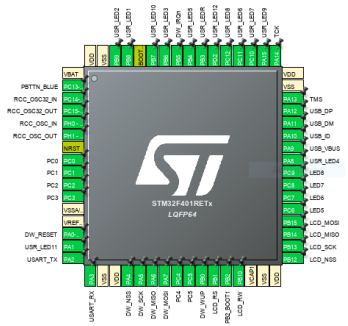

Fig. 3. Changing the pinout of the microcontroller

reads the measured distances and sends them to the $\mathrm{PC}$ via serial communication. For the firmware of this device, the base code was used, which was given with the DWM1000 chip, but some modifications were necessary, consisting of modifying the state machine that drives the DWM1000 chip and creating a serial communication for periodical sending and reading of distance messages.

b) PC Software: A Python script, running on the PC, directly connected to the listener device, is responsible for the position calculation. The corresponding Python script is a threaded code, i.e. it seeks for new messages on a specific serial port on a thread. When new measurements are available, the calculation of the position is executed, which also has its own thread.

The position calculation is done via a numerical method by minimizing a properly defined cost function (see Section III-D). The function receives the distance measurements and the tag's position is obtained by applying the Scipy library's minimize function [17].

\section{SySTEM PROPERTIES}

Several considerations have to be taken to achieve proper positioning accuracy with the given hardware and software configuration, hereinafter we discuss these main points and their role in the positioning process.

\section{A. Communication scheme}

Several methods exist in the literature for determining position [12]. Time Difference of Arrival (TDoA) [5] based approaches use the time differences between the tag-toanchor receive times. This is then converted into distance differences representing hyperboles; their intersection gives the tag's position.

Time Sum of Arrival (TSoA) method is based on reflection of the signal. It measures the time it takes the signal to go from one anchor, reflect on the tag and finally be received on another anchor. This time can be converted into distance that is a sum of two tag-to-anchor distances. The sum of distances represents an ellipse with the two anchors in the focus points [6].

The above methods require a very precise synchronization of the devices' clock. To overcome this demanding requirement, the Time of Flight $(\mathrm{ToF})$ techniques have been proposed [9], based on the simple fact that time between two points can be converted into distance if the propagation speed is known.

Accordingly, in the underlying configuration we have selected the ToF based techniques for the distance computation. However, there are various methods within the ToF based 
framework: One Way, Two Way and Double-Sided Two Way Ranging.

One Way Ranging (OWR) method contains only a single message between the tag and the anchor (see Figure 4) and computes the time of flight as the difference between the transmission $\left(t_{A}\right)$ and reception $\left(t_{B}\right)$ times:

$$
t_{T O F}=t_{B}-t_{A} .
$$

OWR based methods still require clock synchronization,

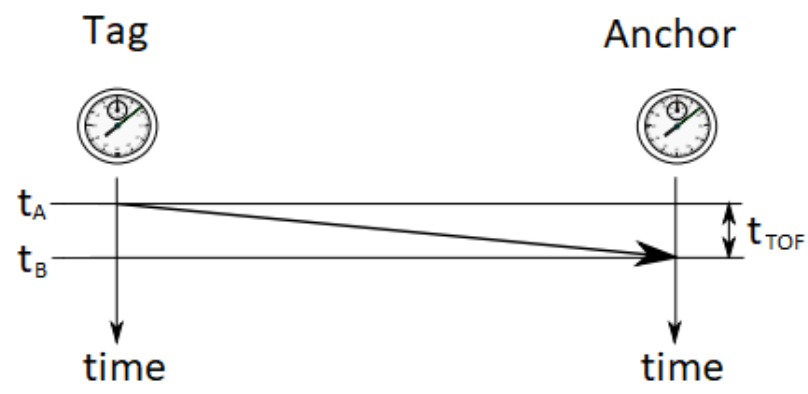

Fig. 4. OWR propagation time measurement

which is eliminated by the Two Way Ranging (TWR) methods, illustrated in Figure 5.

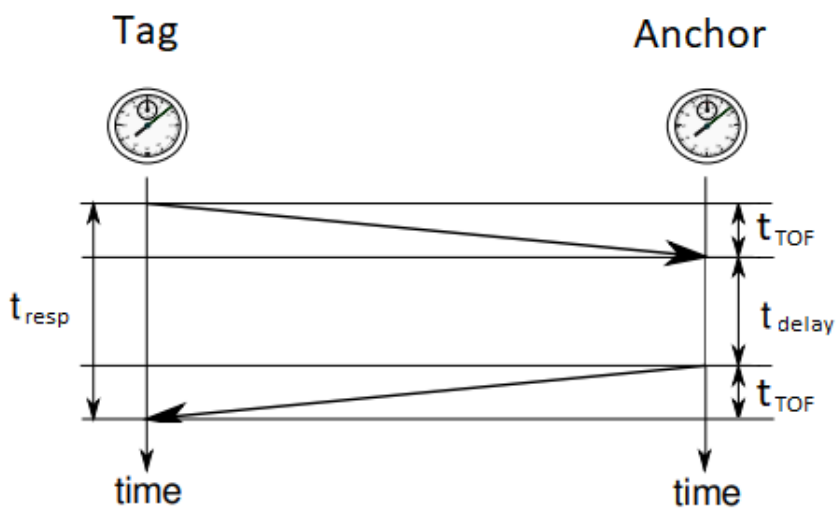

Fig. 5. TWR propagation time measurement

The problem of clock synchronization is solved by adding a second message coming from the anchor to the tag. In the TWR $t_{\text {resp }}$ and $t_{\text {delay }}$ are measured independently on each device and the $t_{T O F}$ can be calculated as:

$$
t_{\text {TOF }}=\frac{t_{\text {resp }}-t_{\text {delay }}}{2} .
$$

The main drawback of this method is that it is half as fast as the OWR. Moreover, if the time measurements are inaccurate (i.e. there is a clock offset), then the speed of time passage differs and consequently a linearly increasing error appears in the measurement. The Symmetrical Double-Sided Two Way Ranging intends to overcome this problem [9].

This method consists of three messages: tag-to-anchor, anchor-to-tag and tag-to-anchor as seen in Figure 6. The error, caused by the clock offset, is reduced by introducing a second TWR scheme, with one of them reversed and one common

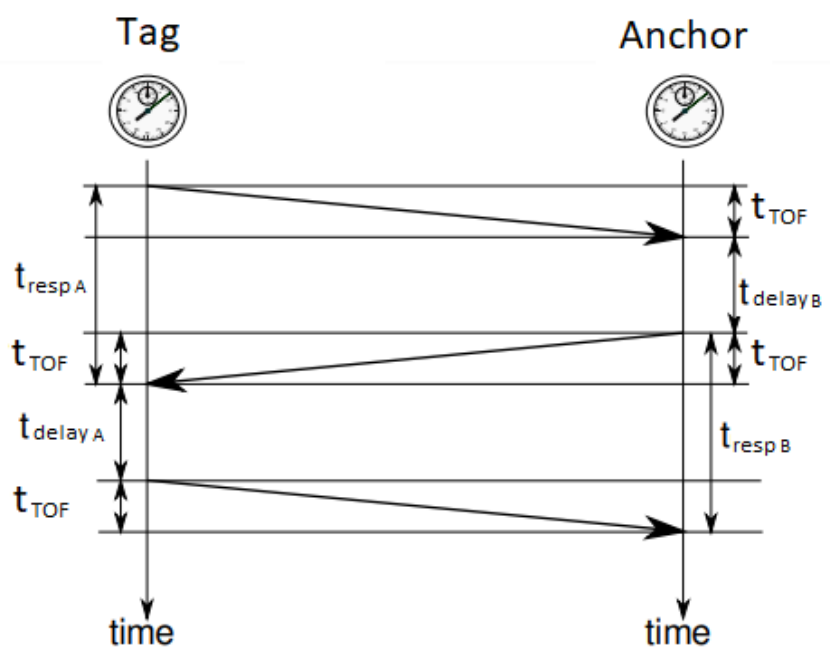

Fig. 6. SDS-TWR propagation time measurement

message in the middle (see Figure 6). Accordingly, the time of flight can be calculated using the four measured times as:

$$
t_{T O F}=\frac{t_{\text {respA }} t_{\text {respB }}-t_{\text {delay } \mathrm{A}} t_{\text {delayB }}}{t_{\text {respA }}+t_{\text {respB }}+t_{\text {delayA }}+t_{\text {delayB }}}[18]
$$

Using these arguments we have selected a ToF based SDS-TWR method for computing the distances between the anchors and the tag. The next arising question is related to the variance of these measurements, which is discussed in the forthcoming sequel.

\section{$B$. Variance of the distance measurements}

UWB signals propagate with the speed of light, therefore a highly accurate transmission- and reception-time measurements is necessary. To illustrate the importance of this, note that a $100 \mathrm{ps}$ error in the time measurement can cause a $3 \mathrm{~cm}$ deviation in the distance. Since perfect time measurement is not possible, the time-of-flight measurements inherits this uncertainty, which can be captured by simple probabilistic models. More precisely if one analyzes the recorded measurements, then it can be seen that they follow a Gaussian distribution (see Figure 7), where the true distance appears as the mean. In order to reduce this measuring uncertainty and

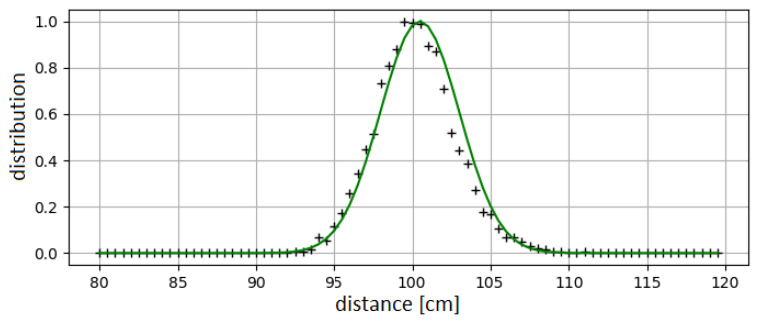

Fig. 7. Variance in the measurements. (+: real, line: matched Gaussian)

increase their reliability, additional actions can be introduced. The simplest solution for this problem is the Moving Average (MA) method, which takes the arithmetic mean of the last $n$ measurements. The closer are the measurements to each 
other in time, the more accurate the approximation will be, therefore this method is useful with more frequent distance updates than necessary.

If the dynamics of the tag is unknown, other more advanced techniques can be applied, such as the Wiener filter, which is a minimum mean square error (MMSE) estimator. However, if some dynamics is given, the popular Kalman filter [19] can be employed.

\section{Kalman Filter}

Kalman filter is a linear quadratic estimator, which gives an optimal estimation of a system's state using the system's dynamics and noisy measurements. One can also use Kalman filter when no known dynamics are at hand by assuming constant state and modelling it with a random walk process. Here linear time-invariant dynamics are assumed:

$$
x_{k}=A x_{k-1}+B u_{k-1}+w_{k-1},
$$

with an also linear observation equation

$$
z_{k}=H x_{k}+v_{k-1} \text {. }
$$

$x_{k}, u_{k}$ and $z_{k}$ are the respective state-, input and output vectors at the discrete-time step $k$, while $w_{k}$ and $v_{k}$ are the process and measurement noise with normal probability distributions. They are assumed to be independent with expected value at zero and known covariance matrices $Q$ and $R$, respectively.

The implementation of the Kalman filter can be split into two parts. The first step is the time update, where the state evolution is predicted one step further using the dynamics:

$$
\begin{gathered}
\hat{x}_{k}^{-}=A \hat{x}_{k-1}+B u_{k-1} \\
P_{k}^{-}=A P_{k-1} A^{T}+Q
\end{gathered}
$$

where $P_{k}$ denotes the covariance of the state estimation error.

In the second part, called measurement update, the predicted state $\left(\hat{x}_{k}^{-}\right)$is corrected with the available measurements information through the Kalman gain $K_{k}$ :

$$
\begin{gathered}
K_{k}=P_{k}^{-} H^{T}\left(H P_{k}^{-} H^{T}+R\right)^{-1} \\
\hat{x}_{k}=\hat{x}_{k}^{-}+K_{k}\left(z_{k}-H \hat{x}_{k}^{-}\right) \\
P_{k}=\left(I-K_{k} H\right) P_{k}^{-}
\end{gathered}
$$

Figure 8 illustrates the effect of the Kalman filter applied on the raw distance measurements of the UWB system. Clearly, the filter narrows the Gaussian distribution and decreases the variance, i.e. a more accurate positioning can be achieved.

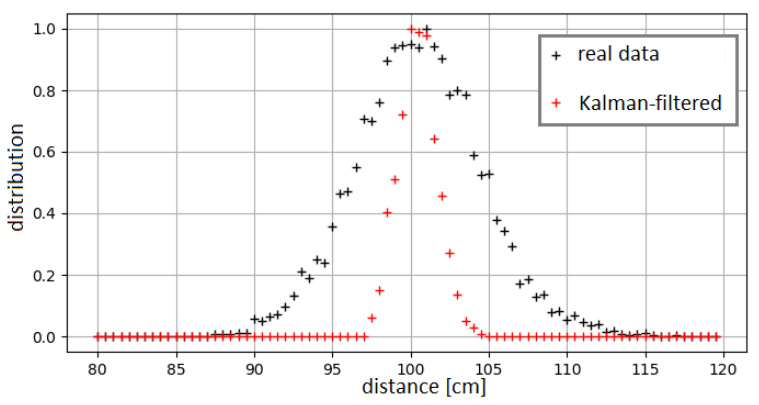

Fig. 8. The Kalman filter's effect on the measurements. (black: real, red: Kalman filtered)
The method has the advantage to cope with the specific dynamics of the tag, whenever it is available. For example the non-linear dynamics of a quad-copter can be incorporated in the same framework by using locally linearized matrices around the actual state estimation $\hat{x}_{k}$, which approach is widely known as Extended Kalman Filter and currently under implementation in our research laboratory.

\section{Position calculation method}

Once an accurate distance calculation is carried out by using the described ToF based SDS-TWR method in combination with the Kalman filtering, the next step is to use these information for computing the position of the tag in a coordinate frame.

For this the anchors' positions (e.g. in 3D coordinates) and the tag's distance from the anchors are available. At least four anchors required to get an unequivocal solution for the position of the tag in 3 dimensions. Three main approaches exist to calculate the position: statistical, algebraic and numerical.

Statistical methods are based on hypothesis about the measurements and on the position of the tag and a probabilistic model which relates these. Due to the nonlinear nature of the problem linear approximations and iterative numerical methods are employed requiring an a priori estimation of the solution to minimize the computation time. The statistical approaches usually classified as open form solvers, providing optimal estimation in case a proper probabilistic model is available [12].

Algebraic methods are based on equations that relates the devices' positions to the distances. These algorithms have the simplest form of models, not requiring a priori information and having a predetermined execution time. The generic equation for these models is the following:

$$
A x+B y+C z=D
$$

where $(x, y, z)$ is the position of the tag, and $\mathrm{A}, \mathrm{B}, \mathrm{C}$ and $\mathrm{D}$ parameters determined by the algorithm (e.g. Pythagorean equations) [12]. Although the algebraic medthods are easy to solve they do not provide an optimal solution.

The third class of algorithms is formed by the numerical methods, which is particularly appealing due to the availability of numerous efficient solvers. Here, we present a simple idea that can be applied easily in the proposed setup, i.e. to minimize a pre-defined cost function:

$$
c(x, y, z)=\sum_{i=1}^{N}\left(\left(x-x_{i}\right)^{2}+\left(y-y_{i}\right)^{2}+\left(z-z_{i}\right)^{2}-d_{i}^{2}\right)^{2},
$$

clearly, this takes its minimum in the idealistic case when the tag's position vector fits completely to the measured distances $\left(d_{i}\right)$ from the anchors (whose coordinates are denoted by the lower index $i$ ). Formally, the position vector $\hat{p}$ is obtained through:

$$
\hat{p}=\underset{x, y, z}{\arg \min } c(x, y, z),
$$

providing optimal solution in realistic setups. This simple optimization based method can be easily implemented through the many available efficient numerical solvers. Alternatively, iterative least square solutions are also applied in positioning problems [20]. 
In order to use the numerical method proposed and outlined above, one needs the coordinates of the anchors, which in fact has a major impact on the problem at hand.

\section{AnChOR Placement And CALIBRATion}

The placement of the anchors significantly influences the accuracy of the position estimation. In order to determine their location, first, simulations were conducted, where the motion of the tag was simulated in a virtual environment and initially all anchors were placed in the same horizontal plane. By analyzing the distribution of the position error this initial arrangement has been modified in several steps, by moving the position of one anchor in each step and evaluate its effect on the accuracy. The final configuration was found to be a tetrahedron, which was then used in the laboratory for installing the anchors.

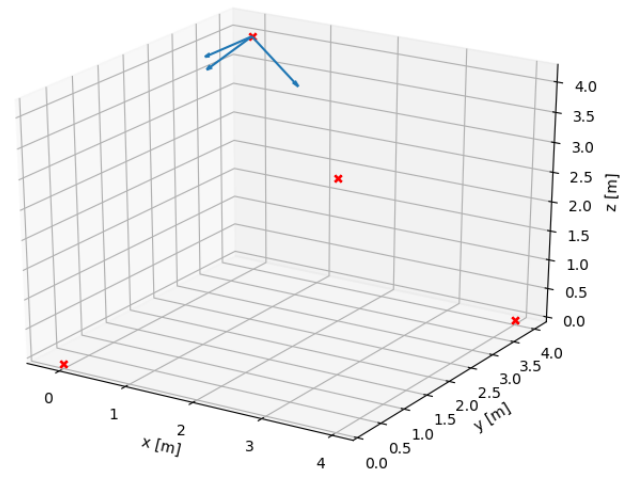

Fig. 9. Anchor positions and the coordinate system choice

When implementing the position estimation in the real environment, a coordinate system has to be chosen. In addition, the locations of the anchors also have to be known and determined in the chosen coordinate frame. According to the best of the knowledge of the authors, no systematic methodology is reported for the initial calibration of ToF based UWB positioning system. Therefore, in order to minimize the number of unknown coordinates the following considerations have been made. First, one can fix the origin of the coordinate system's at the position of one of the anchors (location $(0,0,0)$ ). Then, the orientation of the coordinate frame can be selected in a way that the $x$ axis points towards an other anchor, which consequently nulls out the $y$ and $z$ coordinates of the location for the second anchor $\left(x_{1}, 0,0\right)$. The following step is to rotate the coordinate frame around the $x$ axis in such a way that the third anchor lays in the $x-y$ plane, i.e. the $z$ coordinate of the third anchor is zero $\left(\left(x_{2}, y_{2}, 0\right)\right)$. In this coordinate frame the fourth anchor is represented by three coordinates: $\left(x_{3}, y_{3}, z_{3}\right)$. Following this procedure it is possible to reduce the number of unknown positions from 12 to 6 . For computing these parameters 6 distance measurements are available and the procedure can be done sequentially using simple geometric considerations.

For this purpose the anchors have been coded to take themselves the measurements between each other. In order to capture all the 6 distances, 3 modified SDS-TWR were performed. In the first phase (see Figure 10), anchor 0 sends a poll message, then anchor 1,2 and 3 respond with 3 messages. After all the responses arrived at anchor 0 , the final message transmitted, and the phase is done when the last message is received by all the anchors. The first phase

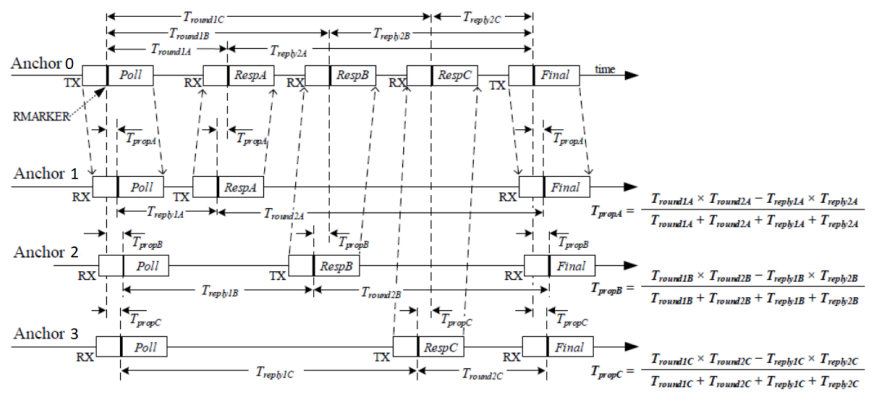

Fig. 10. The first phase of the initial calibration

determines 3 distances out of the 6 : the distances between anchor 0 and the other three. The next phase is started by anchor 1, which sends a poll message to anchor 2 and anchor 3 , to which they respond with 2 response messages (see Figure 11). After anchor 1 received all the responses, the final message is sent, and when anchor 2 and 3 receive this message the second phase is finished. With the second phase,

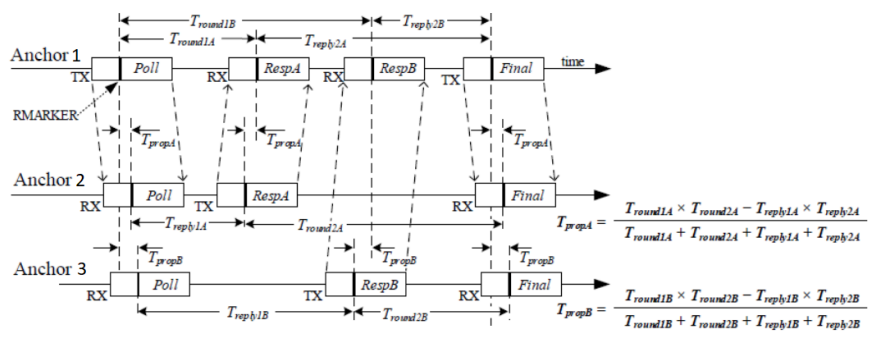

Fig. 11. The second phase of the initial calibration

another 2 distances are known, namely between anchor 1 and 2 , and anchor 1 and 3 . In the third phase, the last distance is measured between the anchor 2 and 3 (Figure 12). When

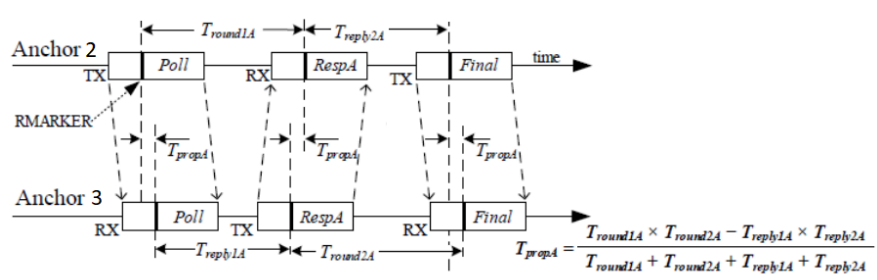

Fig. 12. The third phase of the initial calibration

anchor 3 receives the anchor 2's final message, the third phase has been completed.

Note that due to the variance in the distance measurements, each phase has to be repeated until the average of each measurement of distances change significantly. If the average remains almost the same, we can stop, and the initial calibration is done. 


\section{VALIDATION}

To evaluate a proposed method a simple validation has been performed in simulation and in the real environment as well.

Firstly, simulation-based validation was performed. The distances were generated from known positions and a Gaussian noise was added to them. After that, a cost function optimization was executed and the resulting position was compared to the real position. The tag was simulated to move along a spiral trajectory from the bottom till the top of the room. The average error was found to be $3.09 \mathrm{~cm}$ with standard deviation of $1.29 \mathrm{~cm}$. In the next step, realworld testing was performed. Because no reference system was available (for comparison) the tag was placed in four positions, knowing the distance between each of the points. Then the positions at every point has been estimated by the positioning system and the corresponding distances were derived between the measured positions. These distances can be then compared to the real distances between the locations. Table V shows the numerical comparison, where the average error was $3.76 \mathrm{~cm}$.

\begin{tabular}{|c|c|c|}
\hline Real distance $[\mathrm{cm}]$ & Measured distance [cm] & $\mid$ Error $\mid[\mathrm{cm}]$ \\
\hline 48.49 & 52.5 & 4.01 \\
\hline 55.46 & 52.5 & 2.96 \\
\hline 110.24 & 113.5 & 3.26 \\
\hline 108.68 & 113.5 & 4.82 \\
\hline
\end{tabular}

TABLE I

COMPARISON OF MEASURED AND ESTIMATED DISTANCES

\section{CONCLUSION}

In this paper, the architecture of an indoor positioning system has been presented. There are two main software elements of the system which have to be suited for the purpose of the system. Considerations have been presented on selecting the communication scheme and the position calculation method. The distribution of the measurements has a non-neglectable deviation, therefore accuracy increasing methods are applied, such as averaging or using Kalman filter if a dynamics is available. A simple numerical optimization based position estimating scheme has also been outlined in the paper. Then, an iterative anchor placement has been proposed based on simulation results. The test of the system has been done by distance measurements, showing a $3.76 \mathrm{~cm}$ accuracy.

Future work should be done on some fine-tuning on the parameters in the system, in order to get the best results in the positioning. The implemented method was the SDSTWR, but other techniques can be examined, e.g. TDoA or D-TWR.

\section{ACKNOWLEDGEMENT}

The research reported in this paper was supported by the Higher Education Excellence Program of the Ministry of Human Capacities in the frame of Artificial Intelligence research area of Budapest University of Technology and Economics (BME FIKPMI/FM).

\section{REFERENCES}

[1] I. Kang and H. Nam, "A Centralized Multi-targets Positioning System based on UWB," in 2016 13th International Conference on Ubiquitous Robots and Ambient Intelligence (URAI), Aug 2016, pp. 552-554.

[2] T. Mai. Global Positioning System History. [Online]. Available: https://www.nasa.gov/directorates/heo/scan/communications/policy/ GPS_History.html

[3] A. Alarifi, A. Al-Salman, M. Alsaleh, A. Alnafessah, S. Alhadhrami, M. Al-Ammar, and H. Al-Khalifa, "Ultra Wideband Indoor Positioning Technologies: Analysis and Recent Advances," Sensors, vol. 16, pp. $1-36,052016$

[4] ITU-R. SM.1755 : Characteristics of ultra-wideband technology. [Online]. Available: https://www.itu.int/rec/R-REC-SM.1755-0-200605I/en

[5] B. Yan and L. Xiaochun, "Research on UWB indoor positioning based on TDOA technique," in 2009 9th International Conference on Electronic Measurement Instruments, Aug 2009, pp. 1-167-1-170.

[6] S. Li, J. Hua, G. Zhong, W. Lu, and B. Jiang, "A TSOA based localization algorithm in wireless networks," in 2016 IEEE 11th Conference on Industrial Electronics and Applications (ICIEA), June 2016, pp. 526-530.

[7] S. H. Cho, S. R. Yeo, H. H. Choi, C. Park, and S. J. Lee, "A design of synchronization method for TDOA-based positioning system," in 2012 12th International Conference on Control, Automation and Systems, Oct 2012, pp. 1373-1375.

[8] B. Choi, K. La, and S. Lee, "UWB TDOA/TOA measurement system with wireless time synchronization and simultaneous tag and anchor positioning," in 2018 IEEE International Conference on Computational Intelligence and Virtual Environments for Measurement Systems and Applications (CIVEMSA), June 2018, pp. 1-6.

[9] H. Kim, "Performance Comparison of Asynchronous Ranging Algorithms," in GLOBECOM 2009 - 2009 IEEE Global Telecommunications Conference, Nov 2009, pp. 1-6.

[10] M. Kwak and J. Chong, "A new Double Two-Way Ranging algorithm for ranging system," in 2010 2nd IEEE InternationalConference on Network Infrastructure and Digital Content, Sep. 2010, pp. 470-473.

[11] A. Wang and Y. Song, "Improved SDS-TWR Ranging Technology in UWB Positioning," in 2018 International Conference on Sensor Networks and Signal Processing (SNSP), Oct 2018, pp. 222-225.

[12] I. Mantilla-Gaviria, M. Leonardi, G. Galati, and J. Balbastre, "Localization algorithms for multilateration (MLAT) systems in airport surface surveillance," Signal, Image and Video Processing, vol. 9, pp. 1-10, 012014.

[13] B. Xia, W. Yuan, N. Xie, and C. Li, "A novel statistical manifold algorithm for position estimation," IEEE/CAA Journal of Automatica Sinica, vol. 6, no. 6, pp. 1513-1518, November 2019.

[14] Z. Liu, D. Hu, Y. Zhao, and Y. Zhao, "An algebraic method for moving source localization using TDOA, FDOA, and differential doppler rate measurements with receiver location errors," EURASIP Journal on Advances in Signal Processing, vol. 2019, no. 1, Apr. 2019. [Online]. Available: https://doi.org/10.1186/s13634-019-0621-9

[15] K. Yang, J. An, X. Bu, and G. Sun, "Constrained Total Least-Squares Location Algorithm Using Time-Difference-of-Arrival Measurements," IEEE Transactions on Vehicular Technology, vol. 59, no. 3, pp. 15581562, March 2010.

[16] DECAWAVE. DWM1000 Module. [Online]. Available: https://www.decawave.com/product/dwm1000-module/

[17] T. S. community. scipy.optimize.minimize. [Online]. Available: https://docs.scipy.org/doc/scipy/reference/generated/scipy.optimize. minimize.html

[18] D. Neirynck, E. Luk, and M. McLaughlin, "An alternative double-sided two-way ranging method," in 2016 13th Workshop on Positioning, Navigation and Communications (WPNC), 2016, pp. 1-4.

[19] H. Yin, W. Xia, Y. Zhang, and L. Shen, "UWB-based indoor high precision localization system with robust unscented Kalman filter," in 2016 IEEE International Conference on Communication Systems (ICCS), Dec 2016, pp. 1-6.

[20] D. Gao, A. Li, and J. Fu, "Analysis of positioning performance of UWB system in metal NLOS environment," in 2018 Chinese Automation Congress (CAC), Nov 2018, pp. 600-604. 